\title{
Metabolomics in the Analysis of Inflammatory Diseases
}

\author{
Sabrina Kapoor, Martin Fitzpatrick, Elizabeth Clay, \\ Rachel Bayley, Graham R. Wallace and Stephen P. Young \\ Rheumatology Research Group, School of Immunity \& Infection, \\ College of Medical and Dental Sciences, University of Birmingham \\ United Kingdom
}

\section{Introduction}

Inflammation is a normal and extraordinarily important component of responses to infection and injury. The cardinal features of swelling, redness, stiffness and increasing temperature are strong indicators of the significant changes in tissue metabolism and the ingress of immune cells into the tissues. The increase in blood flow which underlies many of these changes may result in changes to the supply of nutrients and in particular the level of oxygen in the tissues. Inward migration of immune cells, which is also enabled by the increased blood flow, will put further stress on the metabolic environment of the tissues. The activity of macrophages and neutrophils in clearing infection and repairing tissue damage also have significant metabolic consequences particularly because of the production of cytokines and cytotoxic molecules such as reactive oxygen species and reactive nitrogen species, which are required to kill invading organisms. Production of these molecules will consume considerable quantities of oxygen, ATP and NADPH. These antimicrobial agents put considerable stress on host cells in the surrounding and distal tissues and can lead to significant loss of protective metabolites such as glutathione.

Most infections and traumatic injuries are cleared or repaired relatively rapidly and metabolic homoeostasis is soon restored. However, there is a broad range of inflammatory diseases which involve chronic activation of the immune system and, as a result, chronic persistent inflammation. We have been studying the metabolic consequences of chronic inflammatory diseases with the aim of identifying metabolic fingerprints which may provide clues about why the localised tissue disease persists. For example, why in rheumatoid arthritis does persistent inflammation lead to widespread cartilage and joint destruction? However, the metabolic consequences of chronic inflammation are much more widespread than the localised disease and can lead on to important comorbidities such as accelerated atherosclerosis and cardiovascular disease. Metabolomic analysis may be able to distinguish between localised and systemic metabolic consequences of inflammation and provide novel targets for therapeutic intervention in these important human diseases. 


\section{Introduction to inflammatory disease}

An indication of the strong link between inflammation and metabolic processes is seen in cachexia, the loss of cellular mass associated with disease. The discovery of the involvement of tumour necrosis factor- alpha (TNF $\alpha)$ in this process earned it the name 'cachexin'. While $\mathrm{TNF} \alpha$ is now known more generally as a mediator of inflammatory responses, the ability of inflammatory cytokines to have such profound effects on cellular and metabolic processes is informative. Systemic inflammation such as that seen in RA causes changes in metabolism and rheumatoid cachexia is a result of chronic inflammation. This is characterised by the loss of muscle mass and preservation of fat mass (Evans et al., 2008). Classically cachexia is characterised by a low BMI. Muscle wasting is a common feature of RA but low BMI is uncommon as the fat mass is preserved or even increased (Summers et al., 2008). Hence, RA patients may present with either the classic low BMI cachexia (1-13\% of RA population) (Munro \& Capell, 1997) or more frequently, the rheumatoid cachexia (10-20\% of RA with controlled disease and 38\% of patients with active RA) (Engvall et al., 2008, Metsios et al., 2009).

The muscle loss that occurs in rheumatoid cachexia is thought to be due to proinflammatory cytokines such as TNF $\alpha$, IL1 and IL6. TNF promotes proteolysis through the ubiquitinproteasome pathway. There is also some evidence that cytokines may prevent an increase in muscle protein synthesis in response to feeding (anabolic resistance) (Summers et al., 2010). In rheumatoid cachexia the degree of muscle wasting is associated with the disease activity of RA (Summers et al., 2010).

\subsection{The inflammatory process}

An acute inflammatory reaction is characterised by the classic cardinal signs of inflammation: heat, redness, swelling and pain. In experimental settings the temporal relationships oedema, accumulation of leukocytes and accumulation of monocytes and macrophages are well established. These events in self-limited inflammatory reactions are coupled with the release of local factors which prevent further release of leukocytes, which allows resolution (Serhan, 2009). The transition from acute inflammation to chronic inflammation is widely viewed as a result of an excess of pro-inflammatory mediators.

\subsection{Inflammatory mediators}

Cytokines are important regulators of inflammation. Some cytokines such as TNF $\alpha$ and interleukin (IL) 1 promote inflammatory responses by inducing cartilage degradation and promoting a cell-mediated immune response. Other cytokines such as IL-4, IL-10 and IL-13 function mainly as anti-inflammatory molecules (Isomaki \& Punnonen, 1997). Key biological targets that have been identified as being involved in a destructive inflammatory reaction are COX-2, pro-inflammatory interleukins, $\mathrm{TNF} \alpha$, migration inhibition factor, interferon gamma and matrix metalloproteinases (Ivanenkov et al., 2008).

Several inflammatory mediators have been identified which are common to several inflammatory diseases. It has been shown that C-reactive protein (CRP) is secreted by several cell types and is capable of directly activating immune cells. This supports a role for CRP as an active inflammatory mediator which has systemic and local effects (Montecucco \& Mach, 2009). 
White adipose tissue has been shown to secrete several inflammatory mediators called adipokines or adipocytokines. These induce their activities by binding to selective transmembrane receptors. Leptin is the most studied adipocytokine and is thought to have an important role in the inflammatory process (Montecucco \& Mach, 2009).

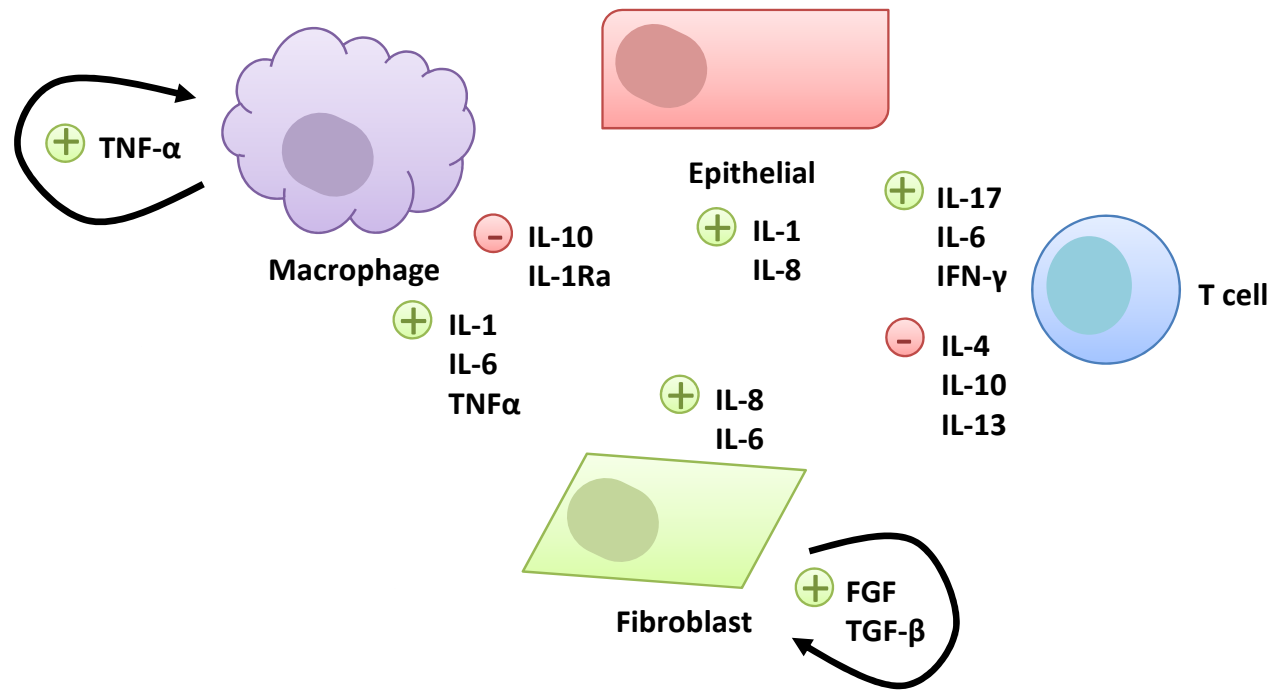

Fig. 1. Key inflammatory cytokines and the inflammatory network. Responses are a balance of pro-inflammatory tumour necrosis factor alpha (TNF $\alpha$ ) and interleukin (IL) 1, IL-6, IL-17 and anti-inflammatory IL-1R, IL-4, IL-10 and IL-13. Expression of cytokines is dependent on activation and local signalling driving progression and eventual resolution.

\subsection{Metabolic inflammation}

Many factors contribute to the complex course of inflammatory reactions. Microbiological, immunological and toxic agents can initiate the inflammatory response by activating a variety of humoral and cellular mediators. In the early phase of inflammation, excessive amounts of interleukins and lipid-mediators are released and play an important role in the pathogenesis of organ dysfunction. Arachidonic acid (AA) is released from membrane phospholipids during inflammatory activation and is metabolised to prostaglandins and leukotrienes. Various strategies have been evaluated to regulate the excessive production of lipid mediators on different levels of biochemical pathways, such as inhibition of phospholipase A2, the trigger enzyme for release of AA, blockade of cyclooxygenase and lipoxygenase pathways and the development of receptor antagonists against platelet activating factor and leukotrienes. Some of these agents exert protective effects in different inflammatory disorders such as septic organ failure, rheumatoid arthritis or asthma, whereas others fail to do so. Encouraging results have been obtained by dietary supplementation with long chain omega-3 fatty acids like eicosapentaenoic acid (EPA). In states of inflammation, EPA is released to compete with AA for enzymatic metabolism inducing the production of less inflammatory and chemotactic derivatives (Heller et al., 1998). 


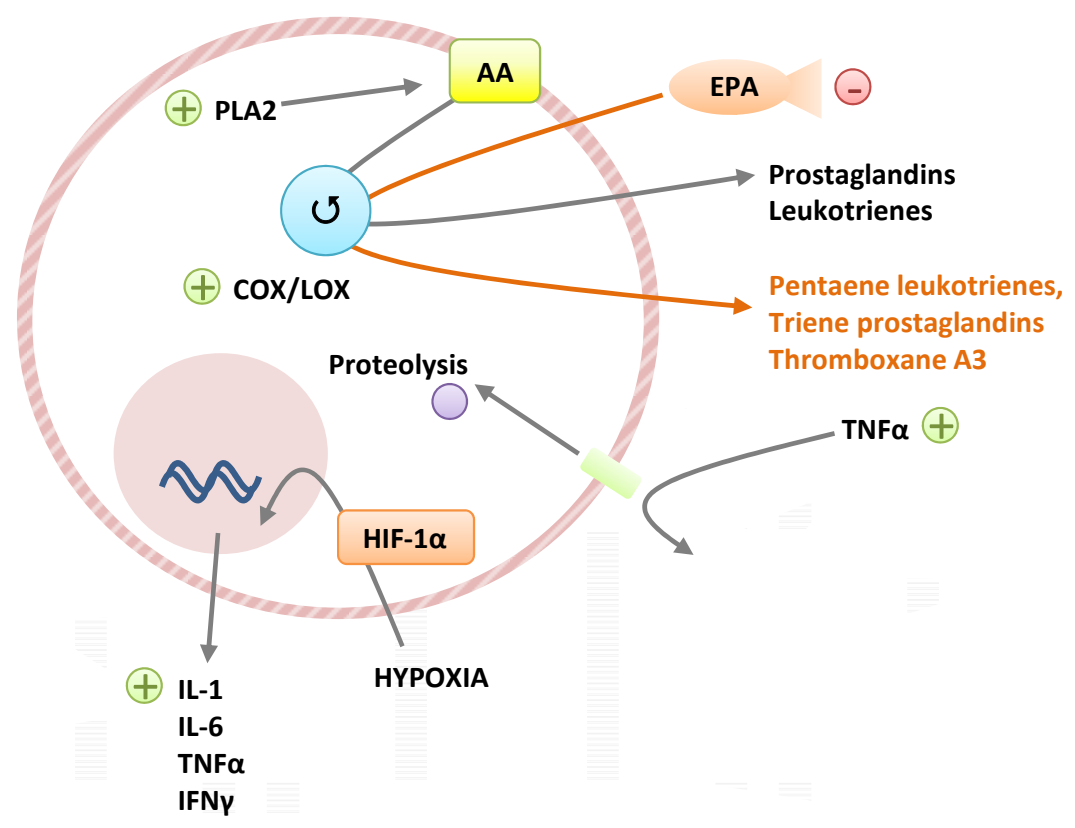

Fig. 2. Some common metabolic responses to inflammation and hypoxia. Arachidonic acid (AA) from cellular membranes is metabolised to inflammatory prostaglandins and leukotrienes. Omega-3 fatty acids (EPA) compete for the same pathway producing less inflammatory derivatives. Hypoxic conditions in the inflammatory site stabilises HIF transcription factor driving production of IL-1, IL-6, TNFa and IFN $\gamma$. TNFa in turn drives cellular proteolysis and tissue remodelling.

When investigating inflammation it is important to take into account the many facets of the inflammatory environment that have the potential to play a role in pathology. Hypoxia is known to be prevalent in the inflammatory environments such as those associated with wounds, malignant tumours, bacterial infections and autoimmunity (Eltzschig \& Carmeliet, 2011, Murdoch et al., 2005). Increasing hypoxia in the inflammatory site is associated with poorer disease outcome such as increased macroscopic synovitis in rheumatoid arthritis $(\mathrm{Ng}$ et al., 2010).

Normal physiological oxygen levels are thought to range between 5-12\% oxygen (compared to $21 \%$ atmospheric oxygen). However, hypoxic tissue oxygen levels in pathological environments can range from as little as $0.5 \%$ oxygen to around $2.5 \%$ oxygen. Local hypoxia develops as the result of either blood vessel occlusion by inflamed tissues, or when existing supply is insufficient for increased cellular density caused by infiltrating or proliferating inflammatory cells. Additionally, circulating phagocytes can block blood vessels reducing blood flow into the inflammatory site (Sitkovsky \& Lukashev, 2005). Normal tissue structures can lend themselves to hypoxia where they are poorly perfused, such as the synovium or eye. Tissue alteration associated with inflammation can contribute to hypoxia by altering pressure within the blood vessels causing vessel occlusion and increasing distances between blood vessels (Jawed et al., 1997, Mapp et al., 1995). 
There is increasing evidence that the inflammatory environment is hypoxic. The tumour environment is known to be hypoxic and extensive angiogenesis reveals the requirement of the tissue for a better oxygen supply. In rheumatoid arthritis, oxygen levels of synovial fluid have been directly measured revealing lower oxygen tensions compared with osteoarthritic patients and patients with traumatic joint injuries (Lund-Olesen, 1970). In systemic sclerosis, direct measurements with sensitive probes revealed lower dermal oxygen levels in fibrotic areas compared to non-fibrotic areas in both patients and healthy controls (Beyer et al., 2009). Metabolomic analysis of eye fluids from uveitis patients has shown increased levels of oxaloacetate and urea, likely derived from anaerobic respiration by locally activated macrophages (Young et al., 2009, Young \& Wallace, 2009).

An elegant cellular oxygen detection system is used by cells to respond to changes in environmental oxygen. Reductions in environmental oxygen lead to the stabilisation of the transcription factor hypoxia-inducible factor (HIF), which is otherwise targeted for depletion in oxygen-rich environments. HIF expression is therefore suggestive of hypoxic exposure, and has been detected in autoimmune diseases such as rheumatoid arthritis and multiple sclerosis (Gaber et al., 2009, Hollander et al., 2001, Lassmann, 2003). HIF is known to be important in inflammatory development, for example loss of HIF-1a in macrophages is associated with impaired aggregation, motility, invasiveness and killing of bacteria (Cramer et al., 2003).

Hypoxia and HIF stabilisation has a large effect on cellular metabolism. HIF causes a preference for glycolytic metabolism over oxidative phosphorylation by inducing the expression of glycolytic enzymes. This allows ATP generation to continue in the absence of sufficient oxygen albeit at a much reduced efficiency per molecule of glucose. It also induces the upregulation of lactate dehydrogenase $\mathrm{A}$, therefore promoting the conversion of pyruvate (produced during glycolysis) to lactate (Wheaton \& Chandel, 2011). Lactate has been detected in many chronic inflammatory conditions such as in inflamed joints (Chang \& Wei, 2011, Treuhaft \& McCarty, 1971), multiple sclerosis , pulmonary inflammation (Serkova et al., 2008) and is thought to play a role in wound healing (Trabold et al., 2003). Conversely, the acidosis associated with increasing lactate concentrations is thought to play a pathogenic role in cell transformation and autoantigen development in some inflammatory environments (Chang \& Wei, 2011). Recently, lactate measurements have been suggested to be useful in the diagnosis of bacterial infections in diabetic foot ulcers compared to noninfected ulcers. Both infected and non-infected ulcers revealed high lactate concentrations, but infected ulcers had significantly higher levels probably due to additional immune and bacterial cell involvement (Loffler et al., 2011). The detection of lactate in metabolomic studies of disease suggests that there may be an inflammatory component, understanding of which may help to direct future treatment.

Immune cells are thought to be highly influenced by hypoxia and HIF stabilisation especially due to the environments they normally act within. In a study performed recently by Gaber et al., peripheral blood CD4+ $\mathrm{T}$ cells placed under hypoxia were found to have a large induction of genes involved in metabolism and homeostasis (Gaber et al., 2009). Innate immune cells such as neutrophils and macrophages are thought to be adapted to function best at lower oxygen tensions as they preferentially use glycolysis to provide ATP even at higher oxygen levels (Cramer et al., 2003). Macrophages are known to accumulate in the hypoxic sites of chronic inflammation (Vergadi et al., 2011), and hypoxia is associated with activation of tissue-resident macrophages. Exposure of macrophages to hypoxic conditions 
is associated with upregulation of a whole gamut of proinflammatory cytokines such as IL-1 (Scannell, 1996), IL-6 (Albina et al., 1995), IFN-ץ (Murata et al., 2002) and TNF- $\alpha$ (White et al., 2004). It is thought that both low oxygen levels and their downstream effects, such as lactate production, may give rise to this macrophage phenotype. That such phenotypic changes are observed in response to the hypoxic conditions of the inflammatory site is strongly suggestive of a role for metabolism in regulation of immune cells. While normal wound resolution is a tightly regulated process, the presence of long-term inflammatory diseases such as rheumatoid arthritis is indicative of the potential for this regulation to go awry. Therefore hypoxia and the resulting change in metabolism may have a profound effect on immune cell behaviour and thus influence disease onset and progression.

Adenosine is another molecule produced in response to hypoxia partly by the hypoxic inhibition of adenosine kinase (Sitkovsky \& Lukashev, 2005). It is difficult to detect due to its local action, but expression of CD39 and CD73, two molecules involved in the extracellular generation of adenosine, provide a marker of its presence in the inflammatory environment. Adenosine can have profound effects on immune cells and is generally perceived to be anti-inflammatory. It is a ligand for specific receptors found on many immune and stromal cells. These receptors are upregulated by hypoxia suggesting hypoxia perpetuates both the production and action of this molecule (Hasko et al., 2008, Sitkovsky \& Lukashev, 2005). These receptors have varying downstream effects, with the expression of the $\mathrm{A} 2 \mathrm{~A}$ associated with the anti-inflammatory disease but the $\mathrm{A} 2 \mathrm{~B}$ receptor expression being implicated in pro-inflammatory conditions such as colitis. Adenosine is known to cause bronchoconstriction when inhaled by asthma and COPD sufferers, but not in healthy controls (Hasko et al., 2008). Higher levels of adenosine A2 receptor are seen in asthma sufferers and these receptors are associated with a pathological role for the molecule in disease (Brown et al., 2008, Hasko et al., 2008).

\subsection{Use of metabolomics in inflammatory diseases}

Systemic inflammation causes changes in metabolism and many studies have investigated individual metabolites in human disease and animal models of inflammation. From these results it is apparent that the levels of many metabolites are altered by the inflammatory process and this has provided insights into the mechanisms of disease and uncovered several potential biomarkers for disease assessment.

Given these profound systemic and localised changes in metabolism provoked by inflammation and inflammatory cytokines, it is not surprising that metabolomics has been used to investigate several inflammatory diseases. Metabolomics is able to assess the changes in several hundred metabolites simultaneously to build disease metabolites profiles. NMR spectroscopy and mass spectrometry have both been used to derive these multiplexed metabolite profiles.

These metabolic "fingerprints" have proven useful in discriminating between different patient groups or identifying responses to therapy, even if the individual metabolites have not been identified. However, identification of sets of specific metabolites can be derived from these fingerprints and this has led to the identification of novel biomarkers and novel pathways in a number of inflammatory diseases. The use of metabolomic analysis of inflammatory diseases will now be discussed in further detail. 


\section{The inflammatory diseases}

\subsection{Aetiology}

Chronic inflammatory diseases exist in many forms, and have the ability to affect many systems of the body. These range from localised areas of inflammation such as the gut in Crohn's disease, to more widespread systemic inflammation as in rheumatoid arthritis (RA). Although the mediators and events leading to chronic inflammation are well characterized, the precise conditions under which acute inflammation becomes chronic are poorly understood. Recent developments have highlighted the importance of genetic factors, environmental influences and the interactions between them in the development of chronic inflammatory disease (Renz et al., 2011).

Research into the genetics of inflammatory disease has been accelerated by genome wide association studies (GWAS), which has allowed identification of genetic mutations associated with an increased risk of developing specific conditions. For example, many immunologically relevant genes have been associated with an increased risk of developing RA. These include human leukocyte antigen (HLA) alleles involved in antigen recognition, and the peptidyl-arginine deiminase type IV (PADI4) gene controlling production of cyclic citrullinated proteins (CCP's) commonly seen in RA (Nishimoto et al., 2010). However, in a complex disease like RA, genetics are not the whole story, as illustrated by the fact that twin studies only report a concordance rate of around 60\% (MacGregor et al., 2000). Thus the importance of external environmental factors in the development of inflammatory diseases should be considered.

Chronic inflammatory diseases have become more prevalent in recent years, and as major genetic changes are unlikely to have occurred over such a short time period, this is likely to be a result of alterations in environmental exposures and lifestyle factors. To date, several factors have been identified as significant contributors including ageing, infection, poor nutrition and smoking.

Smoking raises an individual's risk of developing inflammatory disease considerably. It has numerous effects on the body including activation of the acute inflammatory response and introduction of large amounts of reactive oxygen species (ROS) (Borgerding \& Klus, 2005). It is unclear as to which particular constituent of smoke induces the inflammatory response; however studies have revealed that smoke contains large amounts of lipopolysaccharide (LPS) (Hasday et al., 1999), which could potentially trigger unwanted immune responses seen in chronic inflammatory disease. An increase in ROS is also evident, as indicated by decreased circulating antioxidants found in smokers (Alberg, 2002). This creates a prooxidant environment and increases the likelihood of oxidative damage to important cellular components.

It is not surprising given the complex and varied nature of chronic inflammatory diseases that the observed phenotype is a result of gene-gene and gene-environment interactions. For example, it has been shown in mice with a mutation in the Crohn's disease (CD) susceptibility gene Atg16L1 who become infected with murine norovirus develop a Crohn'slike disease (Stappenbeck et al., 2010). There was no evidence of pathology in the wild type mouse, suggesting the presence of two risk factors is required to induce disease. Another example of gene-environment interactions in disease development was found when looking 
at the interaction between RA susceptibility genes HLA-DRB1 and PTPN22 and their interaction with smoking (Kallberg et al., 2007). It was observed that the odds ratio (OR) of developing RA with two genetic risk factors was 13.2, which rose to 23.4 if two genetic factors were present and there was a history of smoking. These studies provides sound evidence that gene-gene and gene-environment interactions occur, and risk of inflammatory disease greatly increases with the presence of more than one additional risk factor.

\subsection{The gut}

Crohn's disease is a chronic debilitating inflammatory disease of the bowel. The exact aetiology is unknown but is thought to be related to the dysregulation of the immune response towards gut microflora (Strober et al., 2007). Urinary metabolite profiling was carried out on a mouse model of Crohn's disease. These samples were analysed using gas chromatography-mass spectrometry and five key metabolic differences were identified between the Crohn's disease model and controls. This suggested that there are alterations of tryptophan metabolism, fucosylation and fatty acid metabolism in Crohn's disease mice and the authors concluded that fucose and xanthurenic acid could be useful markers of gut inflammation (Lin et al., 2009).

Using a mouse model of inflammatory bowel disease (IBD) to investigate urinary metabolites using NMR, it was found that there was an increase in trimethylamine (TMA) and fucose compared to controls. The increase in TMA was parallel to the progression of IBD (Murdoch et al., 2008). A mouse model of Ulcerative Colitis (UC) was used to looked at serum and urinary metabolites (Schicho et al., 2010). These authors found that both serum and urine were equally powerful for detecting colitis but the metabolites responsible for the differences were different for serum and urine.

Metabolomics of faecal extracts have also been used to study inflammatory bowel disease (Bezabeh et al., 2009). It is sometimes difficult to distinguish Crohn's disease (CD) from UC and some cases are labelled as indeterminate. Over time these cases are usually identified by a combination of endoscopic, radiological and histological techniques. Earlier identification could aid treatment and prognostication. Metabolomic analysis of faecal extracts of patients with both inflammatory diseases showed reduced levels of butyrate, acetate, methylamine and TMA compared to control (Marchesi et al., 2007). Comparing the $\mathrm{UC}$ and CD samples glycerol, alanine, isoleucine, leucine, lysine and valine were present in higher quantities in $\mathrm{CD}$ compared to UC. Acetate was lower in CD compared to UC (Marchesi et al., 2007). Metabolic differences were more marked in CD indicating that inflammation is more extensive in CD compared to UC.

Urinary metabolites have also been used to distinguish CD and UC in humans (Williams et al., 2009). They found that specific urinary metabolites related to gut metabolism differed between $C D$, UC and controls. Hippurate was lowest in $C D$ and differed significantly between CD, UC and controls. Formate levels were higher in CD than in UC or controls and 4-cresol sulphate was lower in CD than in UC or controls (Williams et al., 2009). Hippurate has been shown to be modulated according to gut microbes and this difference is likely to reflect changes in intestinal microbes.

In summary several studies have looked at IBD. The studies have shown that both in mice and in humans TMA is an important marker of IBD (Marchesi et al., 2007, Murdoch et al., 
2008, Schicho et al., 2010). This has been shown using both urine samples or faecal extracts. Hence, TMA may be a useful biomarker for IBD.

\subsection{The eye}

As a closed and immuno-privileged site, the eye provides an ideal system for metabolic analysis. Metabolic products of inflammatory infiltrate accumulate in the vitreous fluid of the eye and may be extracted during other corrective surgery.

Metabolomics has been used to look at vitreous humour in order to differentiate ocular inflammatory diseases (Young et al., 2009). Vitreous fluid samples were taken from patients undergoing retinal surgery and analysed using NMR. Patients had various retinal disorders including chronic non-infectious uveitis (CU), lens-induced uveitis (LIU), proliferative diabetic retinopathy, proliferative vitreoretinopathy (PVR), rhegmatogenous retinal detachment, candida endopthalmitis and varicella zoster virus acute retinal necrosis. The different disease groups showed clear separation using principle component analysis (PCA) and partial least squared discriminate analysis (PLSDA). The majority of the patients had LIU and CU. When looking at LIU and CU specifically there was clear separation and individual metabolites from the spectra showed significant differences with urea, oxaloacetate and glucose all being raised in LIU compared to CU. As urea and oxaloacetate are both involved in the urea cycle it suggests that there is more active inflammation in the LIU patients (Young et al., 2009).

NMR has also been used to look at ocular metabolism in pig eyes (Greiner et al., 1985). They used phosphorous NMR and found phosphorous containing metabolites in aqueous and vitreous fluids (Greiner et al., 1985). In addition to quantifying metabolites, phosphorous NMR can be used to monitor the rate of metabolic change in a specific biochemical reaction and the rate of change in the concentration of a particular metabolite (Greiner et al., 1985). Phosphorous NMR provides a non-invasive method to analyse ocular tissues metabolically and detect subtle biochemical changes that precede manifestations of disease. Such detection may allow for early and more effective therapeutic intervention.

\subsection{Neurological disease}

Multiple sclerosis (MS) is a chronic inflammatory disease affecting the nervous system. Its aetiology is still not completely understood (Ibrahim \& Gold, 2005). It is characterised by demyelination, axonal loss and breakdown of the blood-brain barrier (Trapp et al., 1999). It is a heterogeneous, relapsing and remitting disease. Different treatments have been shown to work at different stages of disease (Rieckmann \& Smith, 2001) so it is important to identify biomarkers that enable identification of different phases.

Interleukin-1 $\beta$ (IL-1 $\beta$ ) and TNF- $\alpha$, have been found to be associated with a broad spectrum of neurological diseases including MS. Griffin et al looked at rat urines to determine whether NMR spectroscopy could detect the presence of IL-1 $\beta$ and TNF- $\alpha$ induced lesions and distinguish between the pathology caused (Griffin et al., 2004). They used an adenoviral vector to induce chronic endogenous expression of either IL-1 $\beta$ or TNF- $\alpha$. They found significant differences between the groups, with the IL-1 $\beta$ treated group showing increases in leucine, isoleucine, valine, n-butyrate and glucose whilst the TNF- $\alpha$ treated group showed increases of citrate, 2-oxoglutarate and succinate (Griffin et al., 2004). 
NMR spectroscopy has also been used to assess cerebrospinal fluid (CSF) in patients with MS. It has been shown that there are increased CSF levels of lactate, creatinine and fructose in MS compared to control patients (Nicoli et al., 1996). Two additional unidentified signals were found to be elevated in MS. The compound responsible for both these signals has now been identified as B-hydroxyisobutyrate (Lutz et al., 2007). This is a typical partial degradation product of branched-chain amino acids. Increased Bhydroxyisobutyrate in urine is thought to be due to respiratory-chain deficiency leading to impaired oxidation of NADH (Chitayat et al., 1992). However the level of Bhydroxyisobutyrate in these experiments was much higher than the level found in CSF from MS patients, and so the precise role of B-hydroxyisobutyrate in MS needs further investigation.

In a study of metabolite fingerprints in the CSF from patients with a range of neurological conditions we have been able to differentiate between some of these conditions by comparing the metabolites found (Sinclair et al., 2010). In particular we were able to identify some novel features of idiopathic intracranial hypertension (IIH) a neurological condition, the pathogenesis of which is poorly understood (Sinclair et al., 2008). Although IIH was not thought to be an inflammatory disease, the elevated levels of lactate we observed in IIH points towards an inflammatory component since lactate has been identified in inflammatory CNS disease previously (Simone et al., 1996). Rabbits with elevated intraocular pressure also show increased levels of lactate which may reflect anaerobic metabolism resulting from decreased blood supply and this may also be an explanation for the lactate in the IIH patients' CSF due to compressed vasculature from the elevated intracranial pressure. Oxaloacetate levels were also increased in IIH and this, together with reduced citrate, suggests alterations in the citric acid cycle. Overall the observations suggest a predominantly anaerobic environment deficient in carbohydrate substrate in patients with $\mathrm{IIH}$, a conclusion supported by the presence of elevated ketone bodies 3-hydroxybutyrate (Sinclair et al., 2010) often observed in hypoxic tissues.

\subsection{Lung disease}

Pulmonary inflammation contributes to the pathogenesis of a number of lung diseases. There is a growing need for validated experimental models that can help our understanding of disease pathogenesis and therapeutic intervention. Traditionally animal models have been used but they have their own problems in representing human disease. Genetic manipulation can greatly enhance animal models. NMR has had some application in the quantification of experimental lung injury.

Serkova et al used Magnetic Resonance Imaging (MRI) and NMR to try and detect and quantify injury in mice following intratracheal administration of inflammatory cytokines (Serkova et al., 2008). Pulmonary inflammation was induced by intratracheal administration of IL-1 $\beta$ and TNF-a. Lung tissue was used for the NMR metabolomics. They showed that with pulmonary inflammation there was a $50 \%$ depletion of ATP and a corresponding elevation of the lactate to glucose ratio suggesting a shift to anaerobic metabolism during inflammation. These returned to control levels at 24 hours (Serkova et al., 2008). These data show that intratracheal administration of IL-1 $\beta$ and TNF- $\alpha$ leads to profound but reversible pulmonary inflammation which is detectable by NMR. 


\subsection{Osteoarthritis}

Osteoarthritis (OA) is a complex disease and has a multifactorial pathogenesis. It has many known risk factors such as age, sex, obesity, activity level, prior joint damage and genetic susceptibility. It is not classically thought of as an inflammatory disease but it may have an inflammatory element. There are currently no disease-modifying drugs for OA and very few are in development.

Synovial fluid (SF) has been used to look at OA via NMR. SF is felt to be a good medium to study as the SF is the first place where the degradation products, enzymes and signal transduction molecules involved in $\mathrm{OA}$ are released from the cartilage matrix. The SF should therefore have a higher concentration of metabolites compared to blood, lymph or urine.

Damayanovich et al used SF from a canine model of OA to look at metabolic profiles using NMR (Damyanovich et al., 1999). Metabolites from experimentally induced canine knee OA SF were compared to metabolites from SF of normal canine knees. They found large increases in lactate and sharp decreases of glucose in OA SF compared to normal SF suggesting that the intra-articular environment of an OA joint is more hypoxic and acidic than a healthy joint. They also found increased levels of pyruvate, lipoprotein associated fatty acids, glycerol and ketones in OA SF suggesting that lipolysis may be an important source of energy in OA. There were also elevated levels of $\mathrm{N}$ acetylglycoproteins, acetate and acetamide in OA SF especially with progressive OA (Damyanovich et al., 1999).

In order to understand further the mechanisms behind OA progression, Damayanovich et al looked at the effect of joint afferent nerve injury (Damyanovich et al., 1999). They again used a bilateral canine model of OA. Paired SF samples were taken from dogs that had undergone bilateral anterior cruciate ligament transaction, unilateral knee denervation and contralateral sham nerve exposure. NMR was used to look at the SF. Increases in glycerol, hydroxybutyrate, glutamine, creatinine, acetate and $\mathrm{N}$-acetyl-glycoprotein were seen in the SF from denervated compared to control knees. This suggests that the metabolite differences seen in the denervated knees are due to the aggravation of OA caused by joint denervation (Damyanovich et al., 1999). Hydroxybutyrate is also found in SF of RA patients (Naughton et al., 1993) suggesting that it is more of a marker of joint destruction rather than being specific for any joint disease.

Another group used guinea pigs to study OA metabolism (Lamers et al., 2003). They used Hartley outbred strain guinea pigs as they develop spontaneous progressive knee OA with features similar to human disease. The earliest histological features appear at 3 months but progress to extensive cartilage degeneration after 12 months. Urine samples were collected from these OA guinea pigs and from healthy animals at 10 and 12 months of age. They identified a metabolic fingerprint that reflected OA changes in the pigs. Lactic acid, malic acid, hypoxanthine and alanine contributed strongly to the fingerprint suggesting their involvement in OA (Lamers et al., 2003). The metabolic profile largely resembled that found in the guinea pig model. The presence of hypoxanthine suggests that OA may be an inflammatory disease due to the increased oxygen demand and altered purine metabolism. 
Mass spectroscopy has also been used to look for novel biomarkers for knee OA (Zhai et al., 2010). They looked at serum samples of unrelated white women with and without knee OA. Knee OA was defined as radiographic, medically diagnosed or total knee replacement due to primary OA. They found that the ratio of valine to histidine and the ratio of leucine to histidine to be significantly associated with knee OA in humans (Zhai et al., 2010). These ratios have potential clinical use as an OA biomarker. OA branched chain amino acids (BCAA) are raised which may drive the release of acetoacetate and 3-hydroxybutyrate. These can result from the partial oxidation of leucine. BCAA are essential amino acids and therefore cannot be synthesised within the body. An increased level of BCAA may suggest an increased rate of protein breakdown or be secondary to collagen degradation. BCAA increase production of the cytokines IL1, IL2, TNF and interferon (Bassit et al., 2000) which could drive the collagen degradation.

\subsection{Rheumatoid arthritis}

Rheumatoid arthritis (RA) is a debilitating systemic inflammatory joint disease. An abnormal metabolic profile in the inflamed joint in RA may be due to the impairment of the vascular supply and/or an increase in the metabolic rate of the inflamed joint.

Hyaluronic acid is a major component of the proteoglycan aggregate of articular cartilage which is required for the functional integrity of extracellular matrix. In RA, SF hyaluronate is depolymerised by the action of reactive oxygen radical species (Parkes et al., 1991). Hyaluronidase activity is absent in both normal and inflamed SF. Generation of reactive oxygen species plays a principal part in synovial hypoxic reperfusion injury (Farrell et al., 1992). This occurs as increased intra-articular pressure during exercise exceeds synovial capillary perfusion pressure leading to impaired blood flow (Mapp et al., 1995).

In 1993, The Inflammation Research Group, The London Hospital Medical College looked at the NMR profiles of RA SF and matched serum samples (Naughton et al., 1993). The NMR profiles of SF were markedly different from their matched serum samples. There were high levels of lactate in the SF compared to the serum and low levels of glucose in the SF compared to the serum. These changes are consistent with the hypoxic status of the rheumatoid joint (Naughton et al., 1993). All the SF samples (RA and control) had lower levels of chylomicron and very-low-density-lipoprotein associated triglycerides compared to their matched serum samples. The SF samples also had high levels of ketone bodies compared to their matched serum samples. These results suggest that the intra-articular environment has an increased utilisation of fats for energy even though it is hypoxic (Naughton et al., 1993, Naughton et al., 1993). They were unable to compare the control SF to the rheumatoid SF due to the low levels of SF aspirated.

Serum from mice has been used to identify a metabolite biomarker pattern associated with RA (Weljie et al., 2007). Using NMR they found that uracil, xanthine and glycine could be used to distinguish arthritic from control animals (Weljie et al., 2007). The presence of the metabolites suggests that nucleic acid metabolism may be highly affected in RA and there may be an association with oxidative stress.

More recently, a group in Denmark have looked at the plasma of patients with RA (Lauridsen et al., 2010). They found differences in the metabolites between patients with RA and healthy controls and differences between patients with active RA and controlled RA. The metabolites 
that they identified were cholesterol, lactate, acetylated glycoprotein and lipids. The lactate levels represented oxidative damage and thus indirectly reflected active inflammation.

\subsection{Atherosclerosis}

Atherosclerosis is the thickening of arteries and is the underlying pathological process that affects the coronary, cerebral, aortic and peripheral arteries. Atherosclerosis involves the accumulation of cholesterol particles, cellular by-products, deposition of the extracellular matrix and inflammatory cell infiltration within the vessel wall (Goonewardena et al., 2010). Chronic inflammation has been recognised as one of the key components of atherogenesis (Ross, 1999) but accelerated atherosclerosis is an important confounder of chronic inflammatory diseases such as rheumatoid arthritis (Bacon et al., 2005). Animal models have been widely used to investigate the biochemical basis of atherosclerosis. Using aortas from apolipoprotein-E knockout mice Mayr et al concluded that inefficient vascular glucose and energy metabolism coincided with increased oxidative stress in animals with hyperlipidaemia (Mayr et al., 2007). NMR-based metabolomics of mouse urine has been used to look at atherosclerosis (Leo \& Darrow, 2009). Using apolipoprotein-E knockout mice they compared untreated mice with those treated with captopril. They found elevated levels of xanthine and ascorbate in untreated mice which may be possible markers of plaque formation (Leo \& Darrow, 2009). The interaction between diet and inflammation in promoting atherosclerosis has also been highlighted through metabolomic studies and Kleenmann (Kleemann et al., 2007) suggested that a high cholesterol intake lead to a switch in liver metabolism towards a pro-atherosclerotic state. Another recent example of how metabolomics can provide novel insights into inflammatory disease pathology was the observation that the metabolism of dietary lecithin by gut flora leads to the increased absorption and accumulation of choline derivatives which in turn promote cardiovascular disease (Wang et al., 2011) . Only through the use of the systematic analysis of metabolites using metabolomics was it possible to uncover these complex metabolic relationships underpinning the disease process.

\section{Conclusion}

As summarised above there is now a growing body of literature describing metabolomic changes in inflammatory diseases, both in humans and animal models. Several distinct metabolic changes have been identified in inflammatory disorders, but there is a core theme of increasing energy requirements coupled with decreasing oxygen supply within the inflammatory environment.

Studies in MS, RA, OA and inflammatory lung disease have all shown an increase in lactate, while studies of inflammatory eye and lung diseases have shown local reductions in glucose. Immunological responses to tissue hypoxia, such as the up-regulation of IL-1, IL-6, IFN- $\gamma$ and TNF- $\alpha$ seen in macrophages, show the link between local metabolic changes and inflammatory responses. Here transcription factor HIF-1a may play a central co-ordinating role in both normal and pathological inflammation by regulating the underlying cellular metabolism towards anaerobic respiratory pathways and lactate production. Subsequent effects of inflammatory cytokines on tissue remodelling and perfusion further provide a mechanism for feedback driving self-sustaining inflammatory microenvironments, and potentially where resolution is disrupted, a route to chronic inflammatory disease. 
Therefore, as both a by-product and mediator of local tissue conditions, metabolites offer a unique opportunity to gain an insight of local and global inflammatory processes. Metabolomics likewise, provides promising opportunities for both diagnosis of inflammatory diseases, and study of the underlying processes that may offer clues as to how the inflammatory process develops.

\section{Acknowledgement}

The authors were supported by grants from Arthritis Research UK (grant numbers 18552 and 19325) and the Wellcome Trust (089384/Z/09/Z and 066490/Z/01/A).

\section{References}

Alberg, A.J. (2002). The influence of cigarette smoking on circulating concentrations of antioxidant micronutrients, Toxicology, vol.180, No. 2, pp.121-37, ISSN 0300-483X.

Albina, J.E., Henry, W.L., Jr., Mastrofrancesco, B., Martin, B.A., Reichner, J.S. (1995). Macrophage activation by culture in an anoxic environment, Journal of Immunology, vol.155, No. 9, pp.4391-6, ISSN 0022-1767.

Bacon, P.A., Church, L.D., Young, S.P. (2005). Endothelial Dysfunction - the Link Between Inflammation and Atherosclerosis in Rheumatoid Arthritis, Journal of the Indian Rheumatology Association, vol.13, pp.103-6, ISSN 0971-5045.

Bassit, R.A., Sawada, L.A., Bacurau, R.F.P., Navarro, F., Rosa, L.F.B.P. (2000). The effect of BCAA supplementation upon the immune response of triathletes, Medicine and Science in Sports and Exercise, vol.32, No. 7, pp.1214-9, ISSN 0195-9131.

Beyer, C., Schett, G., Gay, S., Distler, O., Distler, J.H. (2009). Hypoxia. Hypoxia in the pathogenesis of systemic sclerosis, Arthritis Research and Therapy, vol.11, No. 2, pp.220, ISSN 1478-6362.

Bezabeh, T., Somorjai, R.L., Smith, I.C.P. (2009). MR metabolomics of fecal extracts: applications in the study of bowel diseases, Magnetic Resonance in Chemistry, vol.47, pp.S54-S61, ISSN 0749-1581.

Borgerding, M., Klus, H. (2005). Analysis of complex mixtures - Cigarette smoke, Experimental and Toxicologic Pathology, vol.57, pp.43-73, ISSN 0940-2993.

Brown, R.A., Spina, D., Page, C.P. (2008). Adenosine receptors and asthma, British Journal of Pharmacology, vol.153 Suppl 1, pp.S446-S56, ISSN 0007-1188.

Chang, X., Wei, C. (2011). Glycolysis and rheumatoid arthritis, International Journal of Rheumatic Diseases, vol.14, No. 3, pp.217-22, ISSN 1756-185X.

Chitayat, D., Meaghervillemure, K., Mamer, O.A., Ogorman, A., Hoar, D.I., Silver, K., Scriver, C.R. (1992). Brain Dysgenesis and Congenital Intracerebral Calcification Associated with 3-Hydroxyisobutyric Aciduria, Journal of Pediatrics, vol.121, No. 1, pp.86-9, ISSN 0022-3476.

Cramer, T., Yamanishi, Y., Clausen, B.E., Forster, I., Pawlinski, R., Mackman, N., et al. (2003). HIF-1alpha is essential for myeloid cell-mediated inflammation, Cell, vol.112, No. 5, pp.645-57, ISSN 0092-8674.

Damyanovich, A.Z., Staples, J.R., Chan, A.D.M., Marshall, K.W. (1999). Comparative study of normal and osteoarthritic canine synovial fluid using $500 \mathrm{MHz} \mathrm{H}-1$ magnetic 
resonance spectroscopy, Journal of Orthopaedic Research, vol.17, No. 2, pp.223-31, ISSN 0736-0266.

Damyanovich, A.Z., Staples, J.R., Marshall, K.W. (1999). H-1 NMR investigation of changes in the metabolic profile of synovial fluid in bilateral canine osteoarthritis with unilateral joint denervation, Osteoarthritis and Cartilage, vol.7, No. 2, pp.165-72, ISSN 1063-4584.

Eltzschig, H.K., Carmeliet, P. (2011). Hypoxia and Inflammation REPLY, New England Journal of Medicine, vol.364, No. 20, pp.1977-, ISSN 0028-4793.

Engvall, I.L., Elkan, A.C., Tengstrand, B., Cederholm, T., Brismar, K., Hafstrom, I. (2008). Cachexia in rheumatoid arthritis is associated with inflammatory activity, physical disability, and low bioavailable insulin-like growth factor, Scandinavian Journal of Rheumatology, vol.37, No. 5, pp.321-8, ISSN 0300-9742.

Evans, W.J., Morley, J.E., Argiles, J., Bales, C., Baracos, V., Guttridge, D., et al. (2008). Cachexia: A new definition, Clinical Nutrition, vol.27, No. 6, pp.793-9, ISSN 02615614.

Farrell, A.J., Williams, R.B., Stevens, C.R., Lawrie, A.S., Cox, N.L., Blake, D.R. (1992). Exercise Induced Release of Vonwillebrand-Factor - Evidence for Hypoxic Reperfusion Microvascular Injury in Rheumatoid-Arthritis, Annals of the Rheumatic Diseases, vol.51, No. 10, pp.1117-22, ISSN 0003-4967.

Gaber, T., Haupl, T., Sandig, G., Tykwinska, K., Fangradt, M., Tschirschmann, M., et al. (2009). Adaptation of human CD4+ T cells to pathophysiological hypoxia: a transcriptome analysis, Journal of Rheumatology, vol.36, No. 12, pp.2655-69, ISSN 0315-162X.

Goonewardena, S.N., Prevette, L.E., Desai, A.A. (2010). Metabolomics and atherosclerosis, Current Atherosclerosis Reports, vol.12, No. 4, pp.267-72, ISSN 1534-6242.

Greiner, J.V., Kopp, S.J., Glonek, T. (1985). Phosphorus Nuclear-Magnetic-Resonance and Ocular Metabolism, Survey of Ophthalmology, vol.30, No. 3, pp.189-202, ISSN 00396257.

Griffin, J.L., Anthony, D.C., Campbell, S.J., Gauldie, J., Pitossi, F., Styles, P., Sibson, N.R. (2004). Study of cytokine induced neuropathology by high resolution proton NMR spectroscopy of rat urine, FEBS Letters, vol.568, No. 1-3, pp.49-54, ISSN 0014-5793.

Hasday, J.D., Bascom, R., Costa, J.J., Fitzgerald, T., Dubin, W. (1999). Bacterial endotoxin is an active component of cigarette smoke, Chest, vol.115, No. 3, pp.829-35, ISSN 0012-3692.

Hasko, G., Linden, J., Cronstein, B., Pacher, P. (2008). Adenosine receptors: therapeutic aspects for inflammatory and immune diseases, Nature Reviews Drug Discovery, vol.7, No. 9, pp.759-70, ISSN 1474-1784.

Heller, A., Koch, T., Schmeck, J., van Ackern, K. (1998). Lipid mediators in inflammatory disorders, Drugs, vol.55, No. 4, pp.487-96, ISSN 0012-6667.

Hollander, A.P., Corke, K.P., Freemont, A.J., Lewis, C.E. (2001). Expression of hypoxiainducible factor 1alpha by macrophages in the rheumatoid synovium: implications for targeting of therapeutic genes to the inflamed joint, Arthritis and Rheumatism, vol.44, No. 7, pp.1540-4, ISSN 0004-3591. 
Ibrahim, S.M., Gold, R. (2005). Genomics, proteomics, metabolomics: what is in a word for multiple sclerosis?, Current Opinion in Neurology, vol.18, No. 3, pp.231-5, ISSN 1350-7540.

Isomaki, P., Punnonen, J. (1997). Pro- and anti-inflammatory cytokines in rheumatoid arthritis, Annals of Medicine, vol.29, No. 6, pp.499-507, ISSN 0785-3890.

Ivanenkov, Y.A., Balakin, K.V., Tkachenko, S.E. (2008). New Approaches to the Treatment of Inflammatory Disease Focus on Small-Molecule Inhibitors of Signal Transduction Pathways, Drugs in RED, vol.9, No. 6, pp.397-434, ISSN 1174-5886.

Jawed, S., Gaffney, K., Blake, D.R. (1997). Intra-articular pressure profile of the knee joint in a spectrum of inflammatory arthropathies, Annals of the Rheumatic Diseases, vol.56, No. 11, pp.686-9, ISSN 0003-4967.

Kallberg, H., Padyukov, L., Plenge, R.M., Ronnelid, J., Gregersen, P.K., van der Helm-van Mil, A., et al. (2007). Gene-Gene and Gene-Environment Interactions Involving HLA-DRB1, PTPN22, and Smoking in Two Subsets of Rheumatoid Arthritis, The American Journal of Human Genetics, vol.80, No. 5, pp.867-75, ISSN 0002-9297.

Kleemann, R., Verschuren, L., van Erk, M.J., Nikolsky, Y., Cnubben, N.H.P., Verheij, E.R., et al. (2007). Atherosclerosis and liver inflammation induced by increased dietary cholesterol intake: a combined transcriptomics and metabolomics analysis, Genome Biology, vol.8, No. 9, pp.R200, ISSN 1474-760X.

Lamers, R.J.A.N., DeGroot, J., Spies-Faber, E.J., Jellema, R.H., Kraus, V.B., Verzijl, N., et al. (2003). Identification of disease- and nutrient-related metabolic fingerprints in osteoarthritic guinea pigs, Journal of Nutrition, vol.133, No. 6, pp.1776-80, ISSN 1096-0007.

Lassmann, H. (2003). Hypoxia-like tissue injury as a component of multiple sclerosis lesions, Journal of the Neurological Sciences, vol.206, No. 2, pp.187-91, ISSN 0022-510X.

Lauridsen, M.B., Bliddal, H., Christensen, R., Danneskiold-Samsoe, B., Bennett, R., Keun, H., et al. (2010). (1)H NMR Spectroscopy-Based Interventional Metabolic Phenotyping: A Cohort Study of Rheumatoid Arthritis Patients, Journal of Proteome Research, vol.9, No. 9, pp.4545-53, ISSN 1535-3907.

Leo, G.C., Darrow, A.L. (2009). NMR-based metabolomics of urine for the atherosclerotic mouse model using apolipoprotein-E deficient mice, Magnetic Resonance in Chemistry, vol.47 Suppl 1, pp.S20-S5, ISSN 1097-458X.

Lin, H.M., Edmunds, S.J., Helsby, N.A., Ferguson, L.R., Rowan, D.D. (2009). Nontargeted Urinary Metabolite Profiling of a Mouse Model of Crohn's Disease, Journal of Proteome Research, vol.8, No. 4, pp.2045-57, ISSN 1535-3893.

Loffler, M., Zieker, D., Weinreich, J., Lob, S., Konigsrainer, I., Symons, S., et al. (2011). Wound fluid lactate concentration: a helpful marker for diagnosing soft-tissue infection in diabetic foot ulcers? Preliminary findings, Diabetic Medicine, vol.28, No. 2, pp.175-8, ISSN 1464-5491.

Lund-Olesen, K. (1970). Oxygen tension in synovial fluids, Arthritis and Rheumatism, vol.13, No. 6, pp.769-76, ISSN 0004-3591.

Lutz, N.W., Viola, A., Malikova, I., Confort-Gouny, S., Ranjeva, J.P., Pelletier, J., Cozzone, P.J. (2007). A branched-chain organic acid linked to multiple sclerosis: First identification by NMR spectroscopy of CSF, Biochemical and Biophysical Research Communications, vol.354, No. 1, pp.160-4, ISSN 0006-291X. 
MacGregor, A.J., Snieder, H., Rigby, A.S., Koskenvuo, M., Kaprio, J., Aho, K., Silman, A.J. (2000). Characterizing the quantitative genetic contribution to rheumatoid arthritis using data from twins, Arthritis and Rheumatism, vol.43, No. 1, pp.30-7, ISSN 00043591.

Mapp, P.I., Grootveld, M.C., Blake, D.R. (1995). Hypoxia, Oxidative Stress and RheumatoidArthritis, British Medical Bulletin, vol.51, No. 2, pp.419-36, ISSN 0007-1420.

Marchesi, J.R., Holmes, E., Khan, F., Kochhar, S., Scanlan, P., Shanahan, F., Wilson, I.D., Wang, Y.L. (2007). Rapid and noninvasive metabonomic characterization of inflammatory bowel disease, Journal of Proteome Research, vol.6, No. 2, pp.546-51, ISSN 1535-3893.

Mayr, M., Madhu, B., Xu, Q. (2007). Proteomics and metabolomics combined in cardiovascular research, Trends in Cardiovascular Medicine, vol.17, No. 2, pp.43-8, ISSN 1873-2615.

Metsios, G.S., Stavropoulos-Kalinoglou, A., Panoulas, V.F., Sandoo, A., Toms, T.E., Nevill, A.M., Koutedakis, Y., Kitas, G.D. (2009). Rheumatoid cachexia and cardiovascular disease, Clinical and Experimental Rheumatology, vol.27, No. 6, pp.985-8, ISSN 0392856 X.

Montecucco, F., Mach, F. (2009). Common inflammatory mediators orchestrate pathophysiological processes in rheumatoid arthritis and atherosclerosis, Rheumatology, vol.48, No. 1, pp.11-22, ISSN 1462-0324.

Munro, R., Capell, H. (1997). Prevalence of low body mass in rheumatoid arthritis: Association with the acute phase response, Annals of the Rheumatic Diseases, vol.56, No. 5, pp.326-9, ISSN 0003-4967.

Murata, Y., Ohteki, T., Koyasu, S., Hamuro, J. (2002). IFN-gamma and pro-inflammatory cytokine production by antigen-presenting cells is dictated by intracellular thiol redox status regulated by oxygen tension, European Journal of Immunology, vol.32, No. 10, pp.2866-73, ISSN 0014-2980.

Murdoch, C., Muthana, M., Lewis, C.E. (2005). Hypoxia regulates macrophage functions in inflammation, Journal of Immunology, vol.175, No. 10, pp.6257-63, ISSN 0022-1767.

Murdoch, T.B., Fu, H., MacFarlane, S., Sydora, B.C., Fedorak, R.N., Slupsky, C.M. (2008). Urinary metabolic profiles of inflammatory bowel disease in interleukin-10 genedeficient mice, Analytical Chemistry, vol.80, No. 14, pp.5524-31, ISSN 0003-2700.

Naughton, D., Whelan, M., Smith, E.C., Williams, R., Blake, D.R., Grootveld, M. (1993). An Investigation of the Abnormal Metabolic Status of Synovial-Fluid from Patients with Rheumatoid-Arthritis by High-Field Proton Nuclear-Magnetic-Resonance Spectroscopy, FEBS Letters, vol.317, No. 1-2, pp.135-8, ISSN 0014-5793.

Naughton, D.P., Haywood, R., Blake, D.R., Edmonds, S., Hawkes, G.E., Grootveld, M. (1993). A Comparative-Evaluation of the Metabolic Profiles of Normal and Inflammatory Knee-Joint Synovial-Fluids by High-Resolution Proton NmrSpectroscopy, FEBS Letters, vol.332, No. 3, pp.221-5, ISSN 0014-5793.

Ng, C.T., Biniecka, M., Kennedy, A., McCormick, J., FitzGerald, O., Bresnihan, B., et al. (2010). Synovial tissue hypoxia and inflammation in vivo, Annals of the Rheumatic Diseases, vol.69, No. 7, pp.1389-95, ISSN 0003-4967. 
Ngumah, Q.C., Buchthal, S.D., Dacheux, R.F. (2006). Longitudinal non-invasive proton NMR spectroscopy measurement of vitreous lactate in a rabbit model of ocular hypertension, Experimental Eye Research, vol.83, No. 2, pp.390-400, ISSN 1096-0007.

Nicoli, F., VionDury, J., ConfortGouny, S., Maillet, S., Gastaut, J.L., Cozzone, P.J. (1996). Cerebrospinal fluid metabolic profiles in multiple sclerosis and degenerative dementias obtained by high resolution proton magnetic resonance spectroscopy, Comptes Rendus de l Academie des Sciences Serie Iii-Sciences de la Vie-Life Sciences, vol.319, No. 7, pp.623-31, ISSN 0764-4469.

Nishimoto, N., Sugino, H., Lee, H.M. (2010). DNA microarray analysis of rheumatoid arthritis susceptibility genes identified by genome-wide association studies (vol 12, pg 403, 2010), Arthritis Research and Therapy, vol.12, No. 3, ISSN 1478-6362.

Parkes, H.G., Grootveld, M.C., Henderson, E.B., Farrell, A., Blake, D.R. (1991). Oxidative Damage to Synovial-Fluid from the Inflamed Rheumatoid Joint Detected by H-1Nmr Spectroscopy, Journal of Pharmaceutical and Biomedical Analysis, vol.9, No. 1, pp.75-82, ISSN 0731-7085.

Renz, H., von Mutius, E., Brandtzaeg, P., Cookson, W.O., Autenrieth, I.B., Haller, D. (2011). Gene-environment interactions in chronic inflammatory disease, Nature Immunology, vol.12, No. 4, pp.273-7, ISSN 1529-2908.

Rieckmann, P., Smith, K.J. (2001). Multiple sclerosis: more than inflammation and demyelination, Trends in Neurosciences, vol.24, No. 8, pp.435-7, ISSN 0166-2236.

Ross, R. (1999). Mechanisms of disease - Atherosclerosis - An inflammatory disease, New England Journal of Medicine, vol.340, No. 2, pp.115-26, ISSN 0028-4793.

Scannell, G. (1996). Leukocyte responses to hypoxic/ischemic conditions, New Horizons, vol.4, No. 2, pp.179-83, ISSN 1063-7389.

Schicho, R., Nazyrova, A., Shaykhutdinov, R., Duggan, G., Vogel, H.J., Storr, M. (2010). Quantitative metabolomic profiling of serum and urine in DSS-induced ulcerative colitis of mice by (1)H NMR spectroscopy, Journal of Proteome Research, vol.9, No. 12, pp.6265-73, ISSN 1535-3907.

Serhan, C.N. (2009). Systems approach to inflammation resolution: identification of novel anti-inflammatory and pro-resolving mediators, Journal of Thrombosis and Haemostasis, vol.7, pp.44-8, ISSN 1538-7933.

Serkova, N.J., Van Rheen, Z., Tobias, M., Pitzer, J.E., Wilkinson, J.E., Stringer, K.A. (2008). Utility of magnetic resonance imaging and nuclear magnetic resonance-based metabolomics for quantification of inflammatory lung injury, American Journal of Physiology-Lung Cellular And Molecular Physiology, vol.295, No. 1, pp.L152-L61, ISSN 1040-0605.

Simone, I.L., Federico, F., Trojano, M., Tortorella, C., Liguori, M., Giannini, P., Picciola, E., Natile, G., Livrea, P. (1996). High resolution proton MR spectroscopy of cerebrospinal fluid in MS patients. Comparison with biochemical changes in demyelinating plaques, Journal of the Neurological Sciences, vol.144, No. 1-2, pp.18290, ISSN 0022-510X.

Sinclair, A.B., Viant, M.R., Ball, A.K., Burdon, M.A., Walker, E.A., Stewart, P.M., Rauz, S., Young, S.P. (2010). NMR-Based Metabolomic Analysis of Cerebrospinal Fluid and Serum in Neurological Diseases - A Diagnostic Tool?, NMR in Biomedicine, vol.23, No. 2, pp.123-32, ISSN 1099-1492. 
Sinclair, A.J., Ball, A.K., Burdon, M.A., Clarke, C.E., Stewart, P.M., Cumow, S.J., Rauz, S. (2008). Exploring the pathogenesis of IIH: An inflammatory perspective, Journal of Neuroimmunology, vol.201, pp.212-20, ISSN 0165-5728.

Sitkovsky, M., Lukashev, D. (2005). Regulation of immune cells by local. tissue oxygen tension: Hif1 alpha and adenosine receptors, Nature Reviews Immunology, vol.5, No. 9, pp.712-21, ISSN 1474-1733.

Stappenbeck, T.S., Cadwell, K., Patel, K.K., Maloney, N.S., Liu, T.C., Ng, A.C.Y., et al. (2010). Virus-Plus-Susceptibility Gene Interaction Determines Crohn's Disease Gene Atg16L1 Phenotypes in Intestine, Cell, vol.141, No. 7, pp.1135-64, ISSN 0092-8674.

Strober, W., Fuss, I., Mannon, P. (2007). The fundamental basis of inflammatory bowel disease, Journal of Clinical Investigation, vol.117, No. 3, pp.514-21, ISSN 0021-9738.

Summers, G.D., Deighton, C.M., Rennie, M.J., Booth, A.H. (2008). Rheumatoid cachexia: a clinical perspective, Rheumatology, vol.47, No. 8, pp.1124-31, ISSN 1462-0324.

Summers, G.D., Metsios, G.S., Stavropoulos-Kalinoglou, A., Kitas, G.D. (2010). Rheumatoid cachexia and cardiovascular disease, Nature Reviews Rheumatology, vol.6, No. 8, pp.445-51, ISSN 1759-4790.

Trabold, O., Wagner, S., Wicke, C., Scheuenstuhl, H., Hussain, M.Z., Rosen, N., Seremetiev, A., Becker, H.D., Hunt, T.K. (2003). Lactate and oxygen constitute a fundamental regulatory mechanism in wound healing, Wound Repair and Regeneration, vol.11, No. 6, pp.504-9, ISSN 1524-475X.

Trapp, B.D., Bo, L., Mork, S., Chang, A. (1999). Pathogenesis of tissue injury in MS lesions, Journal of Neuroimmunology, vol.98, No. 1, pp.49-56, ISSN 0165-5728.

Treuhaft, P.S., McCarty, D.J. (1971). Synovial fluid pH, lactate, oxygen and carbon dioxide partial pressure in various joint diseases, Arthritis and Rheumatism, vol.14, No. 4, pp.475-84, ISSN 0004-3591.

Vergadi, E., Chang, M.S., Lee, C., Liang, O.D., Liu, X., Fernandez-Gonzalez, A., Mitsialis, S.A., Kourembanas, S. (2011). Early macrophage recruitment and alternative activation are critical for the later development of hypoxia-induced pulmonary hypertension, Circulation, vol.123, No. 18, pp.1986-95, ISSN 0009-7322.

Wang, Z.N., Klipfell, E., Bennett, B.J., Koeth, R., Levison, B.S., Dugar, B., et al. (2011). Gut flora metabolism of phosphatidylcholine promotes cardiovascular disease, Nature, vol.472, No. 7341, pp.57-65, ISSN 0028-0836.

Weljie, A.M., Dowlatabadi, R., Miller, B.J., Vogel, H.J., Jirik, F.R. (2007). An inflammatory arthritis-associated metabolite biomarker pattern revealed by H-1 NMR Spectroscopy, Journal of Proteome Research, vol.6, No. 9, pp.3456-64, ISSN 1535-3893.

Wheaton, W.W., Chandel, N.S. (2011). Hypoxia. 2. Hypoxia regulates cellular metabolism, American Journal of Physiology - Cell Physiology, vol.300, No. 3, pp.C385-C93, ISSN 0363-6143.

White, J.R., Harris, R.A., Lee, S.R., Craigon, M.H., Binley, K., Price, T., Beard, G.L., Mundy, C.R., Naylor, S. (2004). Genetic amplification of the transcriptional response to hypoxia as a novel means of identifying regulators of angiogenesis, Genomics, vol.83, No. 1, pp.1-8, ISSN 0888-7543.

Williams, H.R.T., Cox, I.J., Walker, D.G., North, B.V., Patel, V.M., Marshall, S.E., et al. (2009). Characterization of Inflammatory Bowel Disease With Urinary Metabolic Profiling, American Journal of Gastroenterology, vol.104, No. 6, pp.1435-44, ISSN 0002-9270. 
Young, S.P., Nessim, M., Falciani, F., Trevino, V., Banerjee, S.P., Scott, R.A.H., Murray, P.I., Wallace, G.R. (2009). Metabolomic analysis of human vitreous humor differentiates ocular inflammatory disease, Molecular Vision, vol.15, No. 125-29, pp.1210-7, ISSN 1090-0535.

Young, S.P., Wallace, G.R. (2009). Metabolomic analysis of human disease and its application to the eye, Journal of Ocular Biology, Disease and Informatics, vol.2, No. 4, pp.235-42, ISSN 1936-8445.

Zhai, G., Wang-Sattler, R., Hart, D.J., Arden, N.K., Hakim, A.J., Illig, T., Spector, T.D. (2010). Serum branched-chain amino acid to histidine ratio: a novel metabolomic biomarker of knee osteoarthritis, Annals of the Rheumatic Diseases, vol.69, No. 6, pp.1227-31, ISSN 0003-4967. 


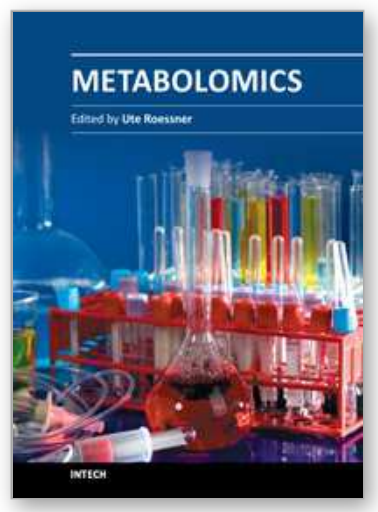

\author{
Metabolomics \\ Edited by Dr Ute Roessner
}

ISBN 978-953-51-0046-1

Hard cover, 364 pages

Publisher InTech

Published online 10, February, 2012

Published in print edition February, 2012

Metabolomics is a rapidly emerging field in life sciences, which aims to identify and quantify metabolites in a biological system. Analytical chemistry is combined with sophisticated informatics and statistics tools to determine and understand metabolic changes upon genetic or environmental perturbations. Together with other 'omics analyses, such as genomics and proteomics, metabolomics plays an important role in functional genomics and systems biology studies in any biological science. This book will provide the reader with summaries of the state-of-the-art of technologies and methodologies, especially in the data analysis and interpretation approaches, as well as give insights into exciting applications of metabolomics in human health studies, safety assessments, and plant and microbial research.

\title{
How to reference
}

In order to correctly reference this scholarly work, feel free to copy and paste the following:

Sabrina Kapoor, Martin Fitzpatrick, Elizabeth Clay, Rachel Bayley, Graham R. Wallace and Stephen P. Young (2012). Metabolomics in the Analysis of Inflammatory Diseases, Metabolomics, Dr Ute Roessner (Ed.), ISBN: 978-953-51-0046-1, InTech, Available from: http://www.intechopen.com/books/metabolomics/metabolomics-inthe-analysis-of-inflammatory-diseases

\section{INTECH}

open science | open minds

\section{InTech Europe}

University Campus STeP Ri

Slavka Krautzeka 83/A

51000 Rijeka, Croatia

Phone: +385 (51) 770447

Fax: +385 (51) 686166

www.intechopen.com
InTech China

Unit 405, Office Block, Hotel Equatorial Shanghai

No.65, Yan An Road (West), Shanghai, 200040, China

中国上海市延安西路65号上海国际贵都大饭店办公楼405单元

Phone: +86-21-62489820

Fax: +86-21-62489821 
(C) 2012 The Author(s). Licensee IntechOpen. This is an open access article distributed under the terms of the Creative Commons Attribution 3.0 License, which permits unrestricted use, distribution, and reproduction in any medium, provided the original work is properly cited. 